\title{
Impaired cerebral autoregulation distal to carotid stenosis/occlusion is associated with increased risk of stroke at cardiac surgery with cardiopulmonary bypass
}

Julia Schoof, MD, ${ }^{a}$ Wiebke Lubahn, MD, ${ }^{a}$ Matthias Baeumer, MD, ${ }^{a}$ Regina Kross, MD, ${ }^{a}$ Claus-Werner Wallesch, MD, Alf Kozian, MD, ${ }^{c}$ Christof Huth, MD, and Michael Goertler, MD ${ }^{\mathrm{a}}$

From the Departments of Neurology, ${ }^{\text {a }}$ Cardiac Surgery, ${ }^{\mathrm{b}}$ and Anesthesia and Intensive Care, ${ }^{\mathrm{C}}$ University of Magdeburg, Magdeburg, Germany.

Received for publication Feb 9, 2007; revisions received March 20, 2007; accepted for publication March 29, 2007.

Address for reprints: Michael Goertler, MD, Department of Neurology, Otto-von-GuerickeUniversität Magdeburg, Leipziger Strasse 44, D-39120 Magdeburg, Germany (E-mail: michael.goertler@medizin.uni-magdeburg. de).

J Thorac Cardiovasc Surg 2007;134:690-6

$0022-5223 / \$ 32.00$

Copyright $\odot 2007$ by The American Association for Thoracic Surgery

doi:10.1016/j.jtcvs.2007.03.018
Objectives: Severe carotid stenosis and occlusion are associated with an increased risk of stroke during and after cardiac surgery with cardiopulmonary bypass. Relevance of an impaired cerebral autoregulation caused by stenosis/occlusion is unknown.

Methods: We prospectively assessed the incidence of stroke in relation to severity of carotid disease and corresponding autoregulatory reserve in 2797 patients who had coronary artery bypass graft and/or valve surgery with cardiopulmonary bypass. Patients underwent preoperative carotid sonography and, in case of severe extracranial disease, transcranial Doppler sonography with carbon dioxide stimulation to assess cerebrovascular reserve capacity.

Results: Sixty-seven (2.4\%) patients had an ischemic stroke, which was fatal in 5. Anterior hemispheric stroke occurred in $42(1.9 \%)$ patients with no/low-grade stenosis, $6(1.8 \%)$ with medium-grade stenosis, $1(0.6 \%)$ with high-grade stenosis/ occlusion and normal autoregulation, and $3(27.3 \%)$ with high-grade stenosis/ occlusion and exhausted autoregulatory reserve. Increased risk was observed in patients with high-grade stenosis/occlusion and exhausted autoregulatory reserve also after adjustment for potential confounders (adjusted odds ratio [OR] 28.3, 95\% confidence interval [CI] 5.8-139.1). Stroke risk was not increased in patients with stenosis/occlusion and normal autoregulation (1.5\%, adjusted OR 0.6, 95\% CI $0.2-1.6)$.

Conclusions: Cerebrovascular reserve capacity evaluated by preoperative transcranial Doppler carbon dioxide testing is a major determinant of stroke risk in patients with carotid artery stenosis/occlusion undergoing cardiac surgery with cardiopulmonary bypass. Its assessment facilitates identification of patients with an excess perioperative stroke risk.

$\mathrm{S}$ troke is a dreaded complication of coronary artery bypass grafting (CABG) and valve surgery with cardiopulmonary bypass (CPB). Incidence is estimated at $2 \%$ and increases to $7 \%$ in combined CABG and valve procedures. ${ }^{1-4}$ The majority of predictors of a perioperative stroke are invariably linked to the patient, such as advanced age, prior stroke, or aortic arch atherosclerosis. ${ }^{5}$ In contrast to these variables, carotid artery stenosis is a modifiable risk factor that has led to suggestions of prophylactic endarterectomy in patients with severe stenosis before or concomitant with cardiac surgery. ${ }^{6}$ Cerebral embolism is considered the most frequent cause of a perioperative stroke. ${ }^{7,8}$ However, embolism from a carotid stenosis triggered by the surgical procedure may be rather unusual-in contrast to embolism from the aortic arch. More likely, carotid stenosis may induce cerebral infarction via hemodynamic compromise during CPB. However, risk of hemodynamic infarction distal to carotid stenosis is linked to impaired cerebral autoregu- 


$$
\begin{aligned}
& \text { Abbreviations and Acronyms } \\
& \begin{aligned}
\mathrm{CABG} & =\text { coronary artery bypass grafting } \\
\mathrm{CI} & =\text { confidence interval } \\
\mathrm{CPB} & =\text { cardiopulmonary bypass } \\
\mathrm{CT} & =\text { computed tomography } \\
\mathrm{OR} & =\text { odds ratio }
\end{aligned}
\end{aligned}
$$

lation. ${ }^{9}$ Therefore, the incidence of perioperative stroke even in severe carotid disease may be increased only in patients with an exhausted cerebral vasodilatative response to low perfusion pressure.

To investigate this hypothesis, we conducted a prospective cohort study, selecting consecutive patients who underwent $\mathrm{CABG}$ or valve surgery with $\mathrm{CPB}$ and had received preoperative carotid sonography including cerebral vasoreactivity testing.

\section{Materials and Methods Patient Selection}

From January 1996 to October 2005, 2797 patients underwent CABG and/or valve surgery with $\mathrm{CPB}$ and had preoperative carotid sonography. Sonography was performed as part of the routine preoperative diagnostics until 1998. From 1999 on, patients were selected on the basis of findings known to be associated with carotid stenosis, such as carotid bruits, prior stroke/transient ischemic attack, and peripheral arterial disease. Patients were excluded from analysis if cardiac surgery was combined with carotid endarterectomy. A total of 377 consecutive cases had to be excluded because their data sets were lost as a result of a change of the electronic case record form in 2002 (Figure 1).

\section{Sonographic Assessment}

Carotid sonography was performed by experienced examiners using a Doppler (Multidop X4, DWL; Elektronische Systeme $\mathrm{GmbH}$, Sipplingen, Germany) and color-duplex device (SSH 380; Toshiba, Tokyo, Japan). Degree of stenosis was quantified according to validated criteria and expressed as local diameter reduction in steps of $10 \%$ according to the method used in the European Carotid Surgery Trial. ${ }^{10-13}$ For the present investigation, stenoses were categorized as none/low grade $(<50 \%)$, medium grade $(50 \%-70 \%)$, high grade $(80 \%-99 \%)$, and occlusion. Patients with high-grade stenosis or occlusion underwent additional transcranial Doppler sonography (Multidop X4, DWL) with carbon dioxide testing to assess cerebrovascular reserve capacity. Mean flow velocity of the middle cerebral artery was measured at rest during normal breathing and after stimulation of cerebral vasodilatation by breathing a mixture of $5 \%$ carbon dioxide and $95 \%$ oxygen. ${ }^{14}$ Because stimulation affects only cerebral resistance vessels, increase of cerebral blood flow correlates with a proportional increase of flow velocity in basal cerebral arteries. An exhausted reserve capacity was assumed at a flow velocity increase of less than 5\% per volume percent increase of end-expiratory carbon dioxide, in-

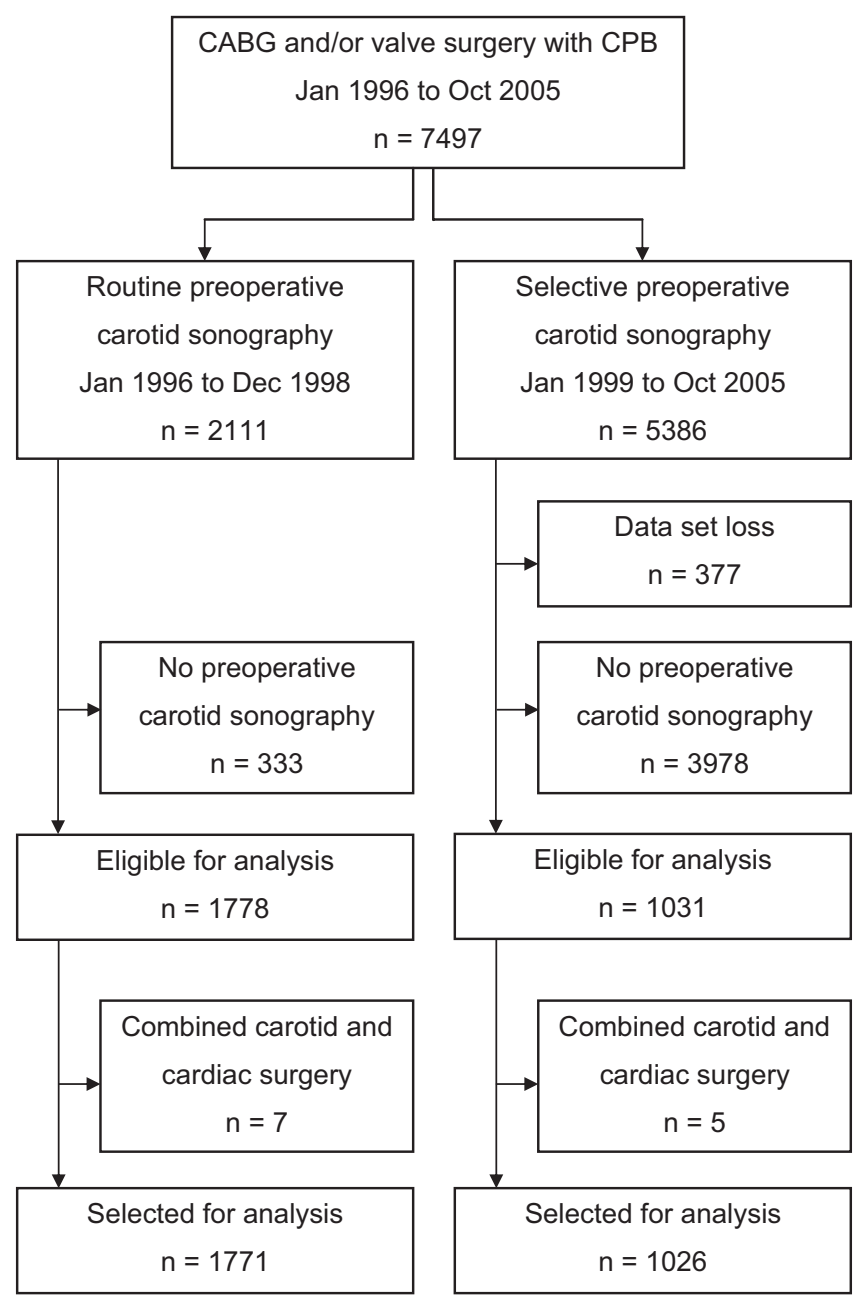

Figure 1. Patient selection. CABG, Coronary artery bypass grafting; $C P B$, cardiopulmonary bypass.

dicating pre-existing autoregulatory vasodilatation owing to stenosis-induced low cerebral perfusion pressure. ${ }^{15}$

\section{Anesthesia and Cardiac Surgery}

Standard anesthesia for cardiac surgery was induced by etomidate $0.2 \mathrm{mg} / \mathrm{kg}$ and sufentanil $1 \mu \mathrm{g} / \mathrm{kg}$ and was maintained with propofol $3 \mathrm{mg} \cdot \mathrm{kg}^{-1} \cdot \mathrm{h}^{-1}$ and with, since 2003 , sevoflurane 0.5 minimal alveolar concentration. Neuromuscular block was achieved by pancuronium $0.08 \mathrm{mg} / \mathrm{kg}$. Standard CPB technique (HL20; Jostra, Hirrlingen, Germany) included nonpulsatile flow, membrane oxygenator, arterial line filter, moderate hypothermia at $29^{\circ} \mathrm{C}$, and alpha-stat method for acid-base management. Heart surgery was performed after administration of cold crystalloid cardioplegic solution (Bretschneider) with a single period of aortic crossclamping. Maintenance of cerebral autoregulation under these conditions has been ascertained. ${ }^{16-22}$

Although the study aimed at evaluating the prognostic relevance of impaired cerebral autoregulation, we considered it unethical with respect to the hypothesized stroke risk not to attempt risk 
reduction in appropriate patients by combined carotid and cardiac surgery; that is, carotid endarterectomy precedes the cardiac procedure in the same operative setting. This strategy was also recommended in patients with severe bilateral stenosis or with contralateral occlusion (ie, high probability of an exhausted cerebrovascular reserve, in whom autoregulation could not be assessed because of insufficient acoustic temporal bone window). During the first study years, combined surgery was also considered in patients with recently symptomatic high-grade carotid stenosis and in patients in whom surgeons judged it adequate to prevent best perioperative stroke, even though transcranial Doppler had demonstrated normal autoregulation. The latter two indications were terminated after literature had demonstrated increased risk of a combined procedure, whereas, according to our own experience, isolated cardiac surgery showed low risk.

\section{Neurologic Outcome Assessment}

Diagnosis of a neurologic outcome event was made by a neurologist who was consulted for suspected signs or symptoms. Patients with focal deficits underwent computed tomographic (CT) scanning to differentiate between ischemic and hemorrhagic events. Ischemic stroke was diagnosed in case of a persisting focal neurologic deficit with compatible CT findings. Postoperative encephalopathy was diagnosed in patients who had prolonged unconsciousness, convulsion, and/or delirium. If the cause remained obscure or neurologic status could not be evaluated sufficiently, CT was performed to exclude or confirm cerebral infarction or bleeding as the underlying disease. An event was considered to be related to the operative procedure if it occurred within 30 days after surgery.

\section{Statistical Analyses}

Statistical analysis was made with the SPSS 11.0 software package (SPSS, Inc, Chicago, Ill). To identify differences in baseline and surgical characteristics between selected patients and those without preoperative carotid sonography, relevant variables were compared by $\chi^{2}$ test and Mann-Whitney $U$ test. The odds ratios (OR) and $95 \%$ confidence intervals (CI) were calculated to compare the incidence of stroke in patients with different degrees of carotid disease and impaired autoregulatory reserve. To evaluate independent contribution of carotid disease and autoregulatory reserve and to adjust for potential confounders, we estimated ORs and $95 \%$ CIs by multivariable logistic regression analysis.

\section{Results}

\section{Patient Characteristics and Sonographic Findings}

Baseline characteristics and surgical data of selected patients versus those who did not undergo preoperative sonography are presented in Table 1. Selected patients exhibited more severe coronary artery disease and more frequent involvement of other organs in the atherosclerotic disease. Patients without preoperative sonography more often underwent urgent/emergency cardiac surgery.

Detailed preoperative sonographic findings are presented in Table 2. Sonographic analysis revealed at least medium- grade stenoses in $528(18.9 \%)$ of 2797 examined patients; $200(7.2 \%)$ patients exhibited high-grade stenosis or occlusion. Eleven patients exhibited unilateral exhausted cerebrovascular reserve capacity distal to an occlusion $(n=9)$ or isolated high-grade stenosis $(n=2)$. In 35 patients with high-grade stenosis/occlusion, carbon dioxide testing could not be performed because of an insufficient acoustic temporal bone window $(n=20)$ or other obstacles $(n=15)$ such as organization failure, unavailability of the device, and cardiopulmonary instability of patients.

\section{Adverse Outcome Events}

Sixty-seven (2.4\%) of the 2797 patients had an ischemic stroke (5 fatal, 62 with persisting deficit) in the anterior $(\mathrm{n}=58)$ or vertebrobasilar circulation $(\mathrm{n}=9)$. Fifteen additional patients $(0.5 \%)$ had focal transient ischemic symptoms, and 2 patients had an intracranial hemorrhage. Sixty-one $(2.2 \%)$ patients had symptoms and findings consistent with an acute encephalopathy. Myocardial infarction occurred in 131 (4.7\%) patients and was fatal in 21. Twenty-five additional patients died of cardiopulmonary insufficiency.

\section{Carotid Stenosis and Outcome}

Incidence of perioperative stroke in relation to carotid disease is presented in Figure 2. Forty-two anterior hemispheric strokes occurred in patients with no/low-grade stenosis, 21 of them more than 24 hours postoperatively in hemodynamically stable patients who had recovered from anesthesia without neurologic deficit. Thirteen of 21 patients whose deficit was diagnosed immediately after recovery from anesthesia revealed anterior hemispheric territorial infarction on subsequent CT scan, suggesting an embolic cause. Stroke was associated with an increased frequency of atrial fibrillation (23.8 vs $8.4 \%$ ) and valve surgery (54.8 vs $27.5 \%$ ). Six patients with medium-grade stenosis had an anterior hemispheric stroke distal to their stenosis, 2 of them more than 48 hours after the operation. One patient with normal cerebrovascular reserve capacity had a stroke distal to a unilateral occlusion on the third postoperative day after he had recovered postoperatively without any neurologic deficit. Three strokes occurred in hemispheres with exhausted cerebrovascular reserve distal to an occlusion (2 patients with bilateral occlusion, 1 with contralateral highgrade stenosis) and were diagnosed immediately after recovery from anesthesia. Three patients who did not undergo preoperative carbon dioxide testing had a stroke distal to unilateral occlusion $(n=2)$ and high-grade stenosis $(n=1)$. Both strokes distal to occlusion showed hemodynamic infarction pattern on CT scans and were diagnosed immediately after recovery from anesthesia, whereas the stroke distal to high-grade stenosis occurred 4 days after the operation. 
TABLE 1. Patient characteristics and surgery data

\begin{tabular}{|c|c|c|c|}
\hline & $\begin{array}{l}\text { Selected CABG/valve operations } \\
\qquad(n=2797)\end{array}$ & $\begin{array}{l}\text { Without preoperative sonography } \\
\qquad(\mathrm{n}=4311)\end{array}$ & $P$ value \\
\hline Age (y) & $64.7 \pm 9.5$ & $64.9 \pm 9.6$ & .364 \\
\hline Female sex & $729(26 \%)$ & $1117(26 \%)$ & .907 \\
\hline \multicolumn{4}{|l|}{ Risk factors } \\
\hline Arterial hypertension & $1941(69 \%)$ & $3596(83 \%)$ & $<.001$ \\
\hline Diabetes & $805(29 \%)$ & $1390(32 \%)$ & .002 \\
\hline Hyperlipidemia & $1772(63 \%)$ & $3090(72 \%)$ & $<.001$ \\
\hline Body mass index $\left(\mathrm{kg} / \mathrm{m}^{2}\right)$ & $27.3 \pm 3.4$ & $27.9 \pm 3.9$ & $<.001$ \\
\hline \multicolumn{4}{|l|}{ History/concomitant diseases } \\
\hline Myocardial infarction & $1405(50 \%)$ & $2004(47 \%)$ & .002 \\
\hline Cardiogenic syncope/shock & $807(29 \%)$ & $894(21 \%)$ & $<.001$ \\
\hline Heart surgery & $106(4 \%)$ & $125(3 \%)$ & .046 \\
\hline Stroke/TIA & $324(12 \%)$ & $119(3 \%)$ & $<.001$ \\
\hline Peripheral vascular disease & $660(24 \%)$ & $461(11 \%)$ & $<.001$ \\
\hline Pulmonary disease & $425(15 \%)$ & $385(9 \%)$ & $<.001$ \\
\hline Renal disease & $493(18 \%)$ & $579(13 \%)$ & $<.001$ \\
\hline \multicolumn{4}{|l|}{ Cardiac function } \\
\hline Atrial fibrillation & $232(8 \%)$ & $366(9 \%)$ & .806 \\
\hline Other arrhythmias & $151(5 \%)$ & $203(5 \%)$ & .211 \\
\hline NYHA classification* & & & .694 \\
\hline Class I/II & $234(8 \%)$ & $455(11 \%)$ & \\
\hline Class III & $2206(79 \%)$ & $3208(74 \%)$ & \\
\hline Class IV & $357(13 \%)$ & $629(15 \%)$ & \\
\hline Surgical urgency & & & $<.001$ \\
\hline Elective & $1531(55 \%)$ & $1487(35 \%)$ & \\
\hline Urgent & $1231(44 \%)$ & $2626(61 \%)$ & \\
\hline Emergency & $35(1 \%)$ & $198(5 \%)$ & \\
\hline Type of surgery & & & .096 \\
\hline CABG & $2050(73 \%)$ & $3246(75 \%)$ & \\
\hline Valve repair/replacement & $394(14 \%)$ & $555(13 \%)$ & \\
\hline Combined procedure & $353(13 \%)$ & $510(12 \%)$ & \\
\hline Diseased coronary vessels $†$ & & & $<.001$ \\
\hline 1 & $335(14 \%)$ & $1003(27 \%)$ & \\
\hline 2 & $680(28 \%)$ & $1365(36 \%)$ & \\
\hline 3 & $1388(58 \%)$ & $1388(37 \%)$ & \\
\hline Aortic valve disease $\ddagger$ & $570(76 \%)$ & $818(77 \%)$ & .848 \\
\hline Mitral valve disease $\ddagger$ & $267(36 \%)$ & $323(30 \%)$ & .018 \\
\hline
\end{tabular}

$C A B G$, Coronary artery bypass grafting; $T I A$, transient ischemic attack. *Twenty patients without sonography not classified. †Related to 2403 and 3756 CABG operations, respectively. $¥$ Related to 747 and 1065 valve operations, respectively.

Three anterior hemispheric strokes in patients with medium-grade $(\mathrm{n}=2)$ and high-grade stenosis $(\mathrm{n}=1)$ occurring in the contralateral hemisphere distal to an unstenosed carotid artery and 9 strokes in the vertebrobasilar circulation were considered unrelated to patients' carotid disease and were excluded from analyses evaluating the relation between the severity of carotid disease and perioperative stroke.

Adjusted odds for categories of different degrees of carotid stenosis in relation to patients with no/low-grade stenosis are presented in Figure 2. The risk of an anterior hemispheric stroke in the flow area of a diseased carotid artery was increased in patients who exhibited an exhausted cerebrovascular reserve capacity distal to high-grade steno- sis or occlusion (OR 19.9, 95\% CI 5.1-77.1). This remained significant after adjustment for age, sex, risk factors, history of myocardial infarction, cardiogenic syncope/shock, cardiac surgery, stroke, and recently symptomatic carotid stenosis, concomitant peripheral arterial disease, atrial fibrillation, New York Heart Association classification, surgical urgency, type of surgery, number of diseased coronary arteries, and type of diseased valves (adjusted OR 28.3, 95\% CI 5.8-139.1). Risk in the 482 patients with carotid disease, that is, at least medium-grade stenosis, and normal cerebrovascular reserve was low (7 strokes, 1.5\%), not exceeding that in patients with no/low-grade stenosis (adjusted OR 0.6, 95\% CI 0.2-1.6). 
TABLE 2. Sonographic findings

\begin{tabular}{lccc}
\hline & All (\% of patients) & $\begin{array}{c}\text { Exhausted autoregulatory } \\
\text { reserve (\% of category) }\end{array}$ & $\begin{array}{c}\text { Autoregulatory reserve not } \\
\text { determined (\% of category) }\end{array}$ \\
\hline Patients & $2797(100 \%)$ & $11(0.4 \%)$ & $35(1.3 \%)$ \\
No/low-grade stenosis & $2269(81.1 \%)$ & & \\
Medium-grade stenosis (unilateral, bilateral) & $328(11.7 \%)$ & $2(7.7 \%)$ & $5(19.2 \%)$ \\
Medium-grade stenosis with contralateral occlusion & $26(0.9 \%)$ & $2(2.1 \%)$ & $16(16.8 \%)^{*}$ \\
High-grade stenosis (unilateral) & $95(3.4 \%)$ & $2(11.1 \%)$ & \\
High-grade stenosis bilateral/with contralateral occlusion & $18(0.6 \%)$ & $3(5.7 \%)$ & $14(26.4 \%)$ \\
Occlusion (unilateral) & $53(1.9 \%)$ & $2(25.0 \%)$ & \\
Occlusion bilateral & $8(0.3 \%)$ & & \\
\hline
\end{tabular}

*Four patients with insufficient acoustic temporal bone window; 12 patients with other obstacles.

Regression analysis selected female sex (OR 2.7, 95\% CI $1.5-4.8)$ and combined coronary and valve surgery (OR 2.6, 95\% CI 1.3-5.3) as additional independent stroke predictors from the above listed covariables. However, when only patients with normal autoregulatory reserve were analyzed, multivariable regression selected female sex, combined coronary and valve surgery, and atrial fibrillation (OR 2.2, 95\% CI 1.1-4.7) as independent predictors but not severity of carotid disease.

Postoperative encephalopathy was not associated with the severity of carotid disease and occurred in $51(2.2 \%)$ patients with no/low-grade stenosis, $9(2.7 \%)$ with mediumgrade stenosis, and $1(0.6 \%)$ with high-grade stenosis/ occlusion and normal autoregulation. No patient with highgrade stenosis/occlusion and an exhausted autoregulatory reserve had encephalopathy.

\section{Combined Carotid and Cardiac Surgery}

Combined surgery was performed in 3 patients with exhausted reserve capacity distal to high-grade stenosis (of whom 1 had an ipsilateral stroke), 2 with bilateral severe disease and untestable autoregulation (1 ipsilateral stroke), 2 with recently symptomatic high-grade stenosis (1 contralateral stroke), and 5 selected on surgeon's decision (1 contralateral stroke).

\section{Discussion}

A recent review yielded a 4-fold risk increase of perioperative stroke at $\mathrm{CABG}$ in patients with significant carotid artery disease. ${ }^{1}$ Stroke incidence increased from $2.0 \%$ in patients with no/low-grade stenosis to $8.4 \%$ in those with at least medium-grade stenosis or occlusion. Maximum risk

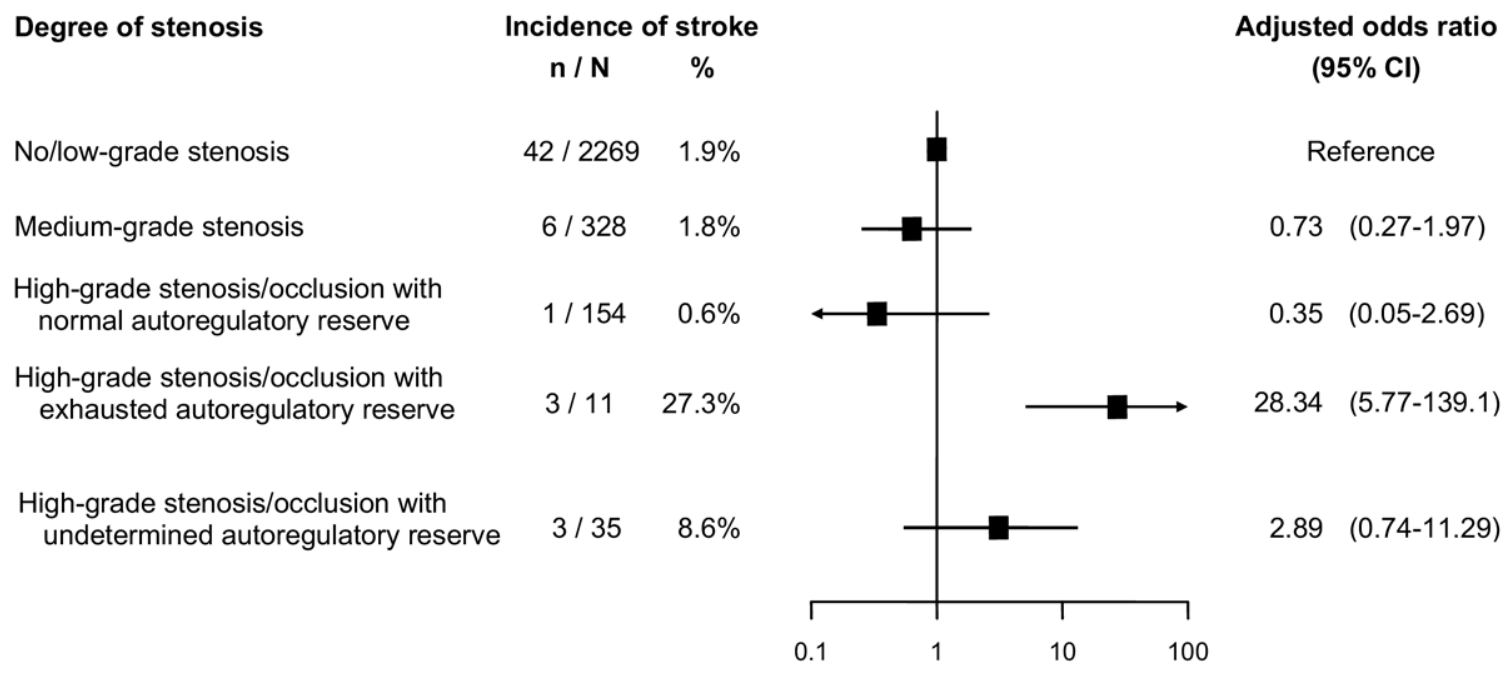

Figure 2. Perioperative stroke related to carotid disease and autoregulatory reserve. Adjusted for age, sex, risk factors, history of myocardial infarction, cardiogenic syncope/shock, cardiac surgery, stroke, and recently symptomatic carotid stenosis, concomitant peripheral arterial disease, atrial fibrillation, New York Heart Association classification, surgical urgency, type of surgery, number of diseased coronary arteries, and type of diseased valves. $\mathrm{Cl}$, Confidence interval. 
was observed distally from an occluded artery with higher risk if both arteries were involved.

Our data suggest an exhausted autoregulatory reserve distal to high-grade carotid stenosis/occlusion as the major determinant of perioperative stroke associated with carotid disease at cardiac surgery. Patients in whom stenosis/occlusion caused lowered cerebral perfusion pressure and induced subsequent cerebral vasodilatation (ie, who had impaired cerebral autoregulation to compensate an additional blood pressure decrease) had a $27 \%$ risk of a perioperative stroke. This represents a nearly 30 -fold excess risk compared with patients with no/low-grade stenosis. In contrast, patients with carotid disease and normal autoregulation exhibited only a $1.5 \%$ incidence of a perioperative stroke, which was not increased compared with patients without carotid disease. However, with respect to the upper limit of the $95 \%$ CI of 1.6, a small risk increase cannot be excluded from our data. Hence, transcranial vasoreactivity testing in patients with high-grade carotid stenosis or occlusion before cardiac surgery may facilitate identification of patients with an excess risk of perioperative stroke by carotid disease.

Both variables found in former studies to be associated with an increased risk of a perioperative stroke-severity of stenosis and bilateralism-were strong predictors of an exhausted cerebrovascular reserve in our series. Risk increase found to be associated with these variables may be caused by patients with an impaired cerebral autoregulation. This also is corroborated by the $8.6 \%$ stroke risk determined in our patients with high-grade stenosis/occlusion who did not undergo hemodynamic testing, which matches the $8.4 \%$ risk determined for patients with comparable vascular findings in the cited review. ${ }^{1}$

Although cerebral embolism is considered the most frequent cause of a perioperative cerebrovascular event, which is corroborated by our study, ${ }^{7,8}$ carotid-originated embolism does not seem to be of relevance in stroke caused by carotid disease at cardiac surgery. Incidence of an ischemic stroke was lower in patients with severe stenosis than in the group with occlusion and was comparable with that in patients without relevant carotid disease when patients with hemodynamic impairment through their stenosis had been excluded.

\section{Clinical Implications}

The incidence of a perioperative stroke associated with carotid stenosis of $50 \%$ or more or occlusion at cardiac surgery with $\mathrm{CPB}$ was low in our series $(2.5 \%, \mathrm{n}=13$, Figure 2). Of these, 4 occurred in patients with isolated occlusion (unilateral/bilateral) not eligible for prophylactic endarterectomy. Seven strokes occurred in patients with medium-grade stenosis (1 distal to contralateral occlusion, 6 in isolated unilateral/bilateral stenosis) with questionable benefit from prophylactic endarterectomy. ${ }^{23}$ Only 2 patients with high-grade stenosis (1 with contralateral occlusion) might have benefited from prophylactic endarterectomy. However, because high-grade stenosis was diagnosed preoperatively in another 111 patients (Table 2), endarterectomy of 113 patients would have been necessary to potentially prevent 2 strokes. Even if carotid endarterectomy could be performed with a risk of perioperative stroke and death as low as in the Asymptomatic Carotid Surgery Trial $(2.8 \%),{ }^{24}$ no net benefit may be expected in this situation by the procedure. In contrast and with respect to the risks estimated from our study, only 4 patients (with proven impaired autoregulation) to 20 patients (without proven normal autoregulation) would have had to be operated on to reach this goal (Table 2). If an undetermined autoregulation can be limited to patients with an insufficient acoustic temporal bone window, the latter figure might be reduced to only 8 patients (Table 2).

Our data suggest that staged or combined endarterectomy of high-grade carotid stenosis before cardiac surgery with CPB is not necessary to prevent stenosis-associated perioperative stroke in patients with normal cerebral autoregulation and probably should not be recommended. Detection of an exhausted cerebrovascular reserve capacity identifies patients who are at high risk for hemodynamic stroke and might benefit from preceding carotid endarterectomy. However, even though we recommended combined surgery in these patients during our observation, this may be an adequate stroke prevention to be recommended only if stroke incidence at combined procedures remains below the $27 \%$ incidence during isolated cardiac surgery established in our study (Figure 2). This cannot be estimated up to date because hemodynamic testing has not been performed in published series and the number of patients with impaired autoregulation undergoing combined operations was far too low at our institution.

However, as an exhausted cerebrovascular reserve is predominantly found distal to occlusion rather than stenosis, nonsurgical precautions may be more effective in this group of patients to prevent perioperative stroke. Blood pressure increase may be the most obvious procedure to increase cerebral blood flow and subsequent oxygen supply in brain regions with impaired autoregulation, although association between blood pressure and incidence of perioperative stroke at cardiac surgery in general is controversially discussed. $^{25}$ Therefore, we intended mean arterial pressure values during $\mathrm{CPB}$ nearby or even above the upper value of the standard 40 to $60 \mathrm{~mm} \mathrm{Hg}$ pressure range in our patients by means of volume substitution and/or vasoconstrictive agents.

\section{Study Limitations}

Baseline characteristics of selected patients differed significantly from those of patients who did not undergo preop- 
erative sonography. However, because we analyzed the effect of carotid disease on cardiac surgery and not its prevalence, pre-selection of patients for sonographic screening on the basis of clinical findings associated with carotid disease seemed reasonable and does not falsify our results. This also may hold true for the series of consecutive patients excluded because of data set loss. An insufficient acoustic temporal bone window, not enabling insonation of the middle cerebral artery in $20(10.0 \%)$ of 200 patients with high-grade stenosis/occlusion, was the most important obstacle for preoperative transcranial carbon dioxide testing. Examination failure in a further $15(7.5 \%)$ patients owing to organization deficiencies, unavailability of the device, and cardiopulmonary instability of patients should be avoidable, at least in part, but may not cause systematic bias. In contrast, bias is introduced by pre-selection of 12 patients with severe stenosis for combined carotid and cardiac surgery. This led to an underestimation of the prevalence of an exhausted autoregulatory reserve in patients with highgrade stenosis (3.5\% vs 5.6\%) and should be respected, particularly when the efficiency of surgical interventions to avoid perioperative stroke is estimated. As we have not investigated aortic arch atherosclerosis, probably the most frequent origin of cerebral embolism at cardiac surgery, and therefore the ratio of cardioembolic stroke may be underestimated in our cohort. However, this does not distort our finding that stroke caused by carotid stenosis is uncommon and may be restricted to patients with an impaired cerebral autoregulation.

In summary, our study suggests that cerebrovascular reserve capacity evaluated by preoperative transcranial carbon dioxide testing is a major determinant of stroke risk in patients with carotid artery stenosis/occlusion subjected to cardiac surgery with CPB. Identification of patients with an exhausted as well as normal reserve capacity may help to improve therapeutic strategies in the presence of combined carotid and cardiac disease.

We thank Jane Heisinger, MT, Steffen Brucks, MD, and all involved colleagues for preoperative sonographic examinations. We thank Siegfried Kropf, PhD, Institute for Biometry and Medical Informatics, University of Magdeburg, for statistical advice.

\section{References}

1. Naylor AR, Mehta Z, Rothwell PM, Bell PRF. Carotid artery disease and stroke during coronary artery bypass: a critical review of the literature. Eur J Vasc Endovasc Surg. 2002;23:283-94.

2. Taylor NE, O'Brian S, Edwards FH, Peterson ED, Bridges CR. Relationship between race and mortality and morbidity after valve replacement surgery. Circulation. 2005;111:1305-12.

3. Salazar JD, Wityk RJ, Grega MA, Borowicz LM, Doty JR, Petrofski JA, et al. Stroke after cardiac surgery: short- and long-term outcomes. Ann Thorac Surg. 2001;72:1195-202.

4. Almassi GH, Sommers T, Moritz TE, Shroyer ALW, London MJ, Henderson WG, et al. Stroke in cardiac surgical patients: determinants and outcome. Ann Thorac Surg. 1999;68:391-8.
5. Das SK, Brow TD, Pepper J. Continuing controversy in the management of concomitant coronary and carotid disease: an overview. Int J Cardiol. 2000;74:47-65.

6. Eagle KA, Guyton RA, Davidoff R, Edwards FH, Ewy GA, Gardner TJ, et al. ACC/AHA 2004 guideline update for coronary artery bypass graft surgery: a report of the American College of Cardiology/American Heart Association Task Force on Practice Guidelines (Committee to Update the 1999 Guidelines for Coronary Artery Bypass Graft Surgery). American College of Cardiology Web Site. Available at: http://www.acc.org/ clinical/guidelines/cabg.pdf 2004

7. Borger MA, Ivanov J, Weisel RD, Rao V, Peniston CM. Stroke during coronary bypass surgery: principal role of cerebral macroemboli. Eur J Cardiothorac Surg. 2001;19:627-32.

8. Hise JH, Nipper ML, Schnitkev JC. Stroke associated with coronary artery bypass grafting. Am J Neuroradiol. 1991;12:811-4.

9. Silvestrini M, Vernieri F, Pasqualetti P, Matteis M, Passarelli F, Troisi $\mathrm{E}$, et al. Impaired cerebral vasoreactivity and risk of stroke in patients with asymptomatic carotid artery stenosis. JAMA. 2000;283:2122-27.

10. de Bray JM, Glatt B. Quantification of atheromatous stenosis in the extracranial internal carotid artery. Cerebrovasc Dis. 1995;5:414-26.

11. Goertler M, Widder B, Schuetz U. Quantifying medium- and highgrade carotid artery stenosis by ultrasound. $J$ Echographie Med Ultrasons. 1996;17:235-9.

12. Görtler M, Niethammer R, Widder B. Differentiating subtotal carotid artery stenoses from occlusions by colour-coded duplex sonography. J Neurol. 1994;241:301-5.

13. European Carotid Surgery Trialists' Collaborative Group. MRC European Carotid Surgery Trial: interim results for symptomatic patients with severe (70-99\%) or with mild (0-29\%) carotid stenosis. Lancet. 1991;337:1235-43.

14. Widder B, Paulat K, Hackspacher J, Mayr E. Transcranial Doppler $\mathrm{CO}_{2}$-Test for the detection of hemodynamically critical carotid artery stenoses and occlusions. Eur Arch Psychiatr Neurol Sci. 1986;236: $162-8$.

15. Kleiser B, Widder B. Course of carotid artery occlusion with impaired cerebrovascular reactivity. Stroke. 1992;23:171-4.

16. Stephan H, Sonntag H, Schenk HD, Kohlhausen S. Effect of Disoprivan (propofol) on the circulation and oxygen consumption of the brain and $\mathrm{CO}_{2}$ reactivity of brain vessels in the human. Anaesthesist. 1987;36:60-5.

17. Gupta S, Heath K, Matta BF. Effect of incremental doses of sevoflurane on cerebral pressure autoregulation in humans. Br J Anaesth. 1997;79:469-72.

18. Stephan H, Groger P, Weyland A, Hoeft A, Sonntag H. The effect of sufentanil on cerebral blood flow, cerebral metabolism and the $\mathrm{CO}_{2}$ reactivity of the cerebral vessels in man. Anaesthesist. 1991;40:153-60.

19. Rozet I, Vavilala MS, Lindley AM, Visco E, Treggiari M, Lam AM Cerebral autoregulation and $\mathrm{CO}_{2}$ reactivity in anterior and posterior cerebral circulation during sevoflurane anesthesia. Anesth Analg. 2006; 102:560-4

20. Patel RL, Turtle MR, Chambers DJ, Venn GE. Effect of differing acid-base regulation on cerebral blood flow autoregulation during cardiopulmonary bypass. Eur J Cardiothorac Surg. 1992;6:302-6.

21. Murkin JM, Farrar JK, Tweed WA, McKenzie FN, Guiraudon G. Cerebral autoregulation and flow/metabolism coupling during cardiopulmonary bypass: the influence of $\mathrm{PaCO}_{2}$. Anesth Analg. 1987;66: 825-32.

22. Preisman S, Marks R, Nahtomi-Shick O, Sidi A. Preservation of static and dynamic cerebral autoregulation after mild hypothermic cardiopulmonary bypass. Br J Anaesth. 2005;95:207-11.

23. Kennedy J, Quan H, Feasby TE, Ghali WA. An audit tool for assessing the appropriateness of carotid endarterectomy. BMC Health Services Res. 2004;4:17-23.

24. MRC Asymptomatic Carotid Surgery Trial (ACST) Collaborative Group. Prevention of disabling and fatal strokes by successful carotid endarterectomy in patients without recent neurological symptoms: randomised controlled trial. Lancet. 2004;363:1491-502.

25. van Wermeskerken GK, Lardenoye JWH, Hill SE, Grocott HP, Phillips-Bute B, Smith PK, et al. Intraoperative physiologic variables and outcome in cardiac surgery: part II. Neurologic outcome. Ann Thorac Surg. 2000;69:1077-83. 